Technical notes

\title{
A new plan quality index for nasopharyngeal cancer SIB IMRT
}

\author{
X. Jin, J. Yi, Y. Zhou, H. Yan, C. Han, C. Xie* \\ Radiotherapy and Chemotherapy Department, The 1st Affiliated Hospital of Wenzhou Medical College, Wenzhou 325000, China
}

\section{A R R T I C L E I N F O}

\section{Article history:}

Received 26 September 2012

Received in revised form

20 March 2013

Accepted 25 March 2013

Available online 16 April 2013

\section{Keywords:}

Nasopharyngeal cancer

SIB-IMRT

Plan quality index

Radiobiological index

\begin{abstract}
A B S T R A C T
A new plan quality index integrating dosimetric and radiobiological indices was proposed to facilitate the evaluation and comparison of simultaneous integrated boost (SIB) intensity modulated radiotherapy (IMRT) plans for nasopharyngeal cancer (NPC) patients.

Ten NPC patients treated by SIB-IMRT were enrolled in the study. Custom software was developed to read dose-volume histogram (DVH) curves from the treatment planning system (TPS). A plan filtering matrix was introduced to filter plans that fail to satisfy treatment protocol. Target plan quality indices and organ at risk (OAR) plan quality indices were calculated for qualified plans. A unique composite plan quality index (CPQI) was proposed based on the relative weight of these indices to evaluate and compare competing plans. Plan ranking results were compared with detailed statistical analysis, radiation oncology quality system (ROQS) scoring results and physician's evaluation results to verify the accuracy of this new plan quality index.

The average CPQI values for plans with OAR priority of low, normal, high, and PTV only were $0.22 \pm 0.08,0.49 \pm 0.077,0.71 \pm 0.062$, and $-0.21 \pm 0.16$, respectively. There were significant differences among these plan quality indices (One-way ANOVA test, $p<0.01$ ). This was consistent with statistical analysis, ROQS results and physician's ranking results in which 90\% OAR high plans were selected.

Plan filtering matrix was able to speed up the plan evaluation process. The new matrix plan quality index CPQI showed good consistence with physician ranking results. It is a promising index for NPC SIBIMRT plan evaluation.
\end{abstract}

(c) 2013 Associazione Italiana di Fisica Medica. Published by Elsevier Ltd. All rights reserved.

\section{Introduction}

Nasopharyngeal cancer (NPC) is endemic in Southern China and part of Southeast Asia. Due to the location of primary tumor surrounded by many critical organs, surgery is usually not feasible for the primary treatment of NPC. Radiotherapy has become the most important treatment modality for NPC.

Intensity modulated radiotherapy (IMRT) has been accepted as a primary treatment modality in the treatment of NPC because of its dose painting ability [1,2]. Rapid dose falloff around the target volume generated by IMRT enables to give higher dose to gross tumor while sparing normal tissues, such as parotid glands, spinal cord, and brainstem. Accelerated radiation therapy with simultaneous integrated boost (SIB) technique may further increase the tumor control probability by reducing the overall treatment time [3]. SIB-IMRT allows for concurrent dose escalation to the primary tumor with multiple prescription doses during optimization. This,

\footnotetext{
* Corresponding author. No.2 Fuxue Lane, Wenzhou 325000, China. Tel.: +86 577 88069316; fax: +8657788069370.

E-mail address: billy07@wzhospital.cn (C. Xie).
}

at the same time, makes treatment planning more difficult and plans more complex.

The selection of an "optimal plan" from competing SIB-IMRT treatment plans for NPC is a daunting task. Currently, plan evaluation mainly relies on visual inspection of the isodose distributions and the dose-volume histograms (DVH). A plan is deemed satisfactory if certain normal tissue dose criteria are met and the isodose lines indicating "good" target coverage. However, this evaluation technique can be ambiguous, since, in many cases, one can generate multiple plans using different objective functions that may be deemed satisfactory. For identical objective functions, SIB-IMRT plans with different OAR (organ at risk) priority could also be generated. This makes ranking SIB-IMRT plans even more important in order to chose the optimal SIB-IMRT plans for NPC patients. For these reasons, traditional methods of evaluating and ranking treatment plans may be too limited for SIB-IMRT plans. This creates the need for an independent dose-volume index to rank SIB-IMRT treatment plans that are generated using different or identical dose-volume constraints.

Since the emergence of 3D treatment planning technique based on CT image data, many quantitative indices have been postulated to describe quantitatively the quality of a given plan [4]. However, 
these parameters usually were all derived from a single reference dose that was normally related to the prescription dose. The effect of different dose to targets and critical organs on the final indices was not demonstrated. Radiobiological parameters had also been suggested for plan evaluation and comparison, but usually as a separated compensate index [5]. The calculation of radiobiological indices is based on a given dosimetric plan and prescribed dose/ fraction. This makes biological and dosimetric indices are correlated and complimentary for plan quality evaluation. With the development of IMRT and other modern radiotherapy techniques, various optimization algorithms of high quality had been applied to create satisfactory plans. As a result, this demands a good plan quality evaluation index for radiotherapy, and motivates efforts to search for good plan quality indices [6]. A new quality plan index was proposed in this study taking both the target coverage and OAR sparing, and both biological parameters TCP (tumor control probability) and NTCP (normal tissue complication probability) into account.

\section{Materials and methods}

\section{Treatment planning}

Ten NPC patients with various stages were enrolled in this study. Target and normal tissue contours have been reported in our previous study and generalized here [7]. Gross tumor volume (GTV) was delineated as the mass shown in the enhanced CT images and/ or MRI images, including the nasopharyngeal tumor, retropharyngeal lymphadenopathy, and enlarged neck nodes. The clinical target volume (CTV), consisting of two parts, CTV1 and CTV2, was defined as the GTV plus a margin of potential microscopic spread. CTV1 encompasses the inferior sphenoid sinus, clivus, skull base, nasopharynx, ipsilateral parapharyngeal space, and posterior third of the nasal cavity and maxillary sinuses. The neck nodes of level 1, 2, 3, 5 were also included. CTV2 encompasses the contralateral parapharyngeal space and neck nodes of level 1, 2, 3, 5. One typical contour was shown in Fig. 1. The planning target volume (PTV) was created based on each volume with an additional $2 \mathrm{~mm}$ margin, allowing for setup variability.

SIB-IMRT technique was applied to generate multi-isocenter plans using BrainSCAN IMRS system, version 5.2 (BrainLAB AG, Heimstetten, Germany) and a small multileaf collimator (MLC) M3 delivery system, as reported in our previous study [8]. Prescription dose to GTV and PTV was $2.5 \mathrm{~Gy}$ and 2 Gy per fraction to a total dose of $70 \mathrm{~Gy}$ and $56 \mathrm{~Gy}$ in 28 fractions, respectively. Normal tissues of brainstem, spinal cord, and parotids were constrained for optimization. Identical optimization parameters and OAR constraints were applied for different OAR priority plan optimization. Four different OAR prioritization plans, namely OAR low, OAR Normal, OAR high, and PTV only, were generated, corresponding to a priority of low, medium, high, and no priority of OARs, respectively [9]. OAR priority was defined by a dynamically changing penalization parameter in the Dynamically Penalized Likelihood algorithm. The penalization parameter defined in the OAR region only. For PTV only plan, the penalization parameter was zero. The larger the penalization parameter, the higher the priority of the OARs becomes, ranging from low to high [10].

\section{DVH data reading}

DVH represents a frequency distribution of average dose values over a 3D matrix of voxels, which composed of targets or organ at risks (OARs) within the patient anatomy. It has been regarded as a strong tool for three-dimensional treatment plan evaluation and a very useful tool for rival plan comparison $[11,12]$. A custom built software (written with MATLAB 7.1) was developed to import and recalculate the DVH data from the BrainSCAN treatment planning system (TPS). Different matrices were built to store the recalculated data for the sake of data analysis. In the GTV and CTV matrices, items include maximum dose (Dmax), minimum dose (Dmin), mean dose (Dmean), volume receiving $93 \%, 95 \%, 110 \%$ and $115 \%$ of the prescribed dose [V93, V95, V110, V115]. The matrix of critical structures, such as brainstem, and spinal cord, includes the maximum dose and mean dose, if the maximum dose larger than its TD50/5 (tolerance dose that would result in 50\% complication probability within 5 years) constraint dose, the volume of this critical structure receiving this constraint dose were also included. The maximum and mean doses of both parotids were reported, also reported were the volume of each parotid that received $26 \mathrm{~Gy}$, and 32 Gy [V26, V32] [13].

\section{Filtering process}

Clinically, there are usually some institute protocols and physician's specific requirements for certain disease and patients. Prior to the participation to plan evaluation and rival plan comparison, every plan will go through a filtering process. This filtering process can filter some plans that fail to meet the protocols or requirements, and save some time and facilitate the comparison process. The following criteria were suggested for the filtering process in this study:
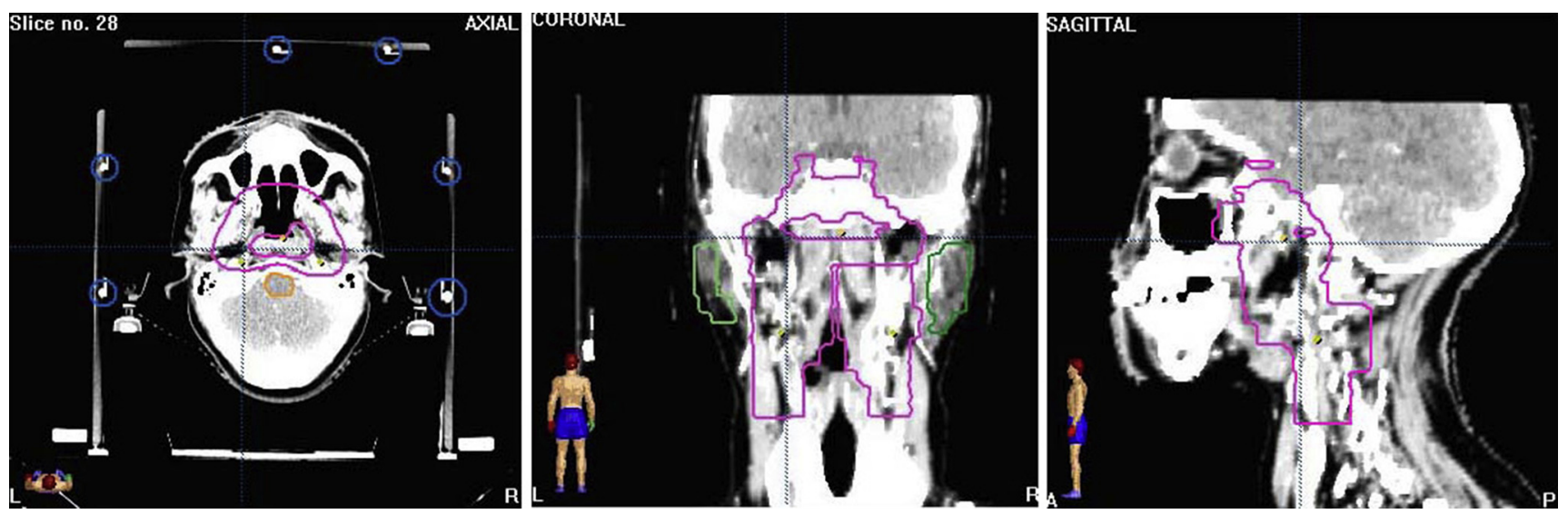

Figure 1. Typical multiple gross tumor volume (GTV) and clinical target volume (CTVs) contour diagram for nasopharyngeal cancer. 
[V93 $<1 \%$, V110 < 20\%, V115 < 5\%] for GTV and CTVs. Dmax $<54 \mathrm{~Gy}$ and $<45 \mathrm{~Gy}$ for brainstem and spinal cord, respectively. V60 $<1 \%$ or $1 \mathrm{cc}$ for brainstem, and V50 $<1 \%$ or $1 \mathrm{cc}$ for spinal cord. D50 $<30 \mathrm{~Gy}$ (dose to $50 \%$ of the volume) for parotids [14]. The constraint priority of these filtering items could be institute and/or case dependent. In this study, the maximum dose constraint on brainstem and cord was given the highest priority. The cold spot constraint item V93 was given a higher priority than those for hot spot constraint, V110 and V115.

\section{Plan evaluation and rival plan comparison}

The following indices were calculated from the extracted DVH for plans passed the filter process for further evaluation and comparison.

Target coverage (TC) of GTV and PTVs was calculated:

$\mathrm{TC}=\frac{V_{\mathrm{T}, \mathrm{Pi}}}{V_{\mathrm{T}}}$

where $V_{\mathrm{T}, \mathrm{Pi}}$ is the target volume that covered by the prescribed dose, which was $95 \%$ of the prescribed dose in this study. The minimum coverage of CTVs, V93 was also calculated for CTV1 and CTV2.

A homogeneity index (HI) similar to that defined in ICRU 62 report was adapted for CTV1 and CTV2 [15],
$\mathrm{HI}=\frac{\mathrm{V} 95-\mathrm{V} 110}{\mathrm{~V} 95}$

Radiobiological ranking index tumor control probability (TCP) and normal tissue complication probability (NTCP) were calculated from the extracted DVH data based on Niemierko model [16].

The equivalent uniform dose (EUD) was calculated by

$\operatorname{EUD}=\left(\sum_{1}^{N} v_{i} D_{i}^{a}\right)^{1 / a}$

where, $N$ is the number of voxels in the structure of interest, $D_{i}$ is the dose in the $i$ th voxel $v_{i}$ and $a$ is the tumor normal tissue-specific parameter that describes the dose-volume effect. $a=-13$ was used for NPC cases [17]. Based on the equivalent uniform dose, the TCP can be calculated by Niemierko [18]

$\mathrm{TCP}=\frac{1}{1+\left[\frac{\mathrm{TCD}_{50}}{\mathrm{EUD}}\right] 4 \gamma_{50}}$

where $\mathrm{TCD}_{50}$ is the tumor dose required to produce $50 \% \mathrm{TCP}$ and $\gamma_{50}$ is the slope of dose response at 50\% TCP. For NPC patients, tumor-specific parameters for head and neck squamous cell (macroscopic) were cited from the study of Okunieff et al. $\left(\mathrm{TCD}_{50}=51.77 \mathrm{~Gy}, \gamma_{50}=2.28\right)[19]$.
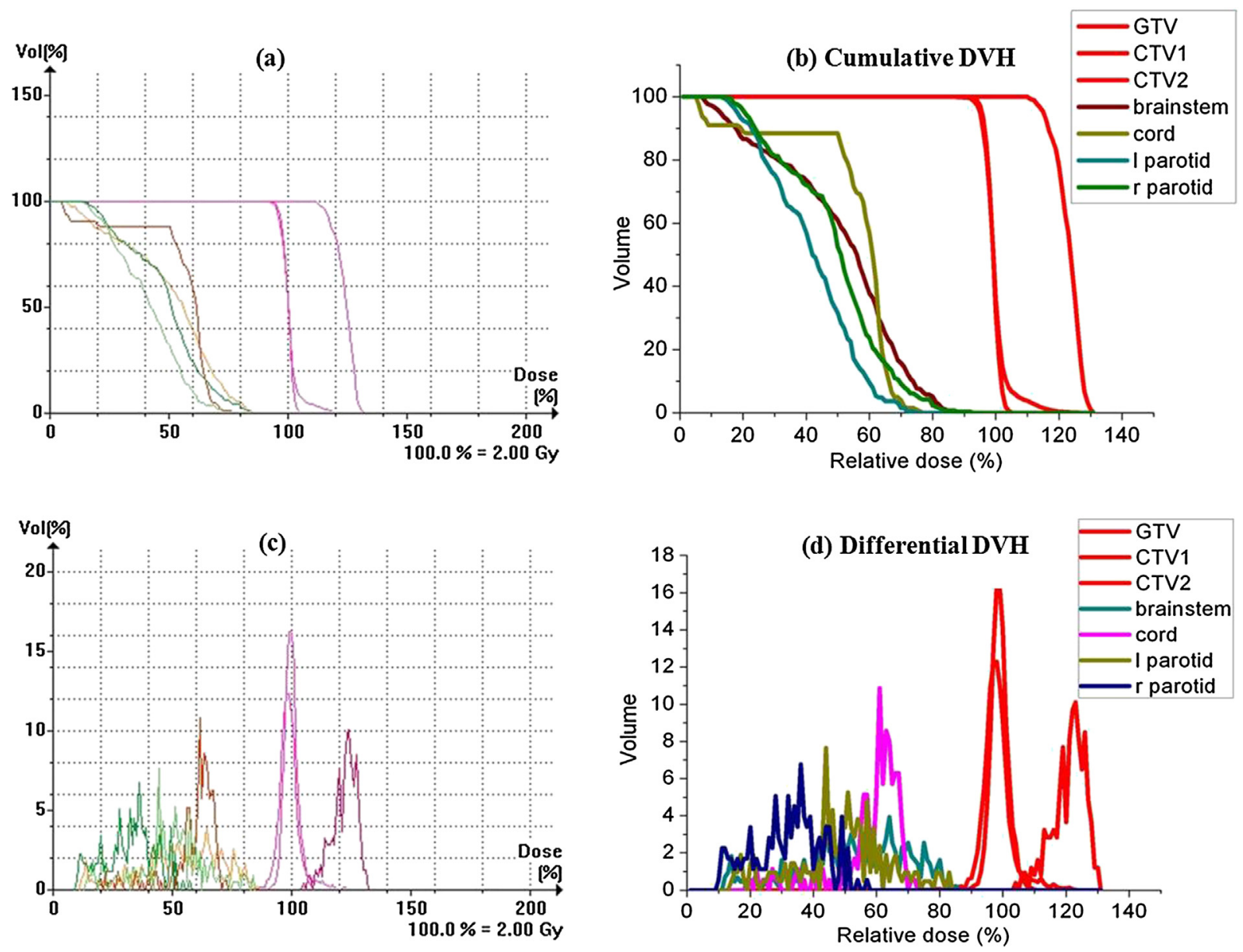

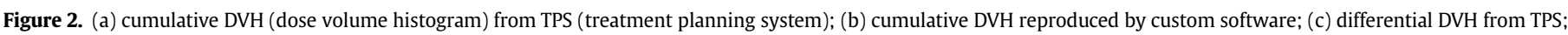
(d) reproduced differential DVH. 
The NTCP was determined as

$$
\mathrm{NTCP}=\frac{1}{1+\left[\frac{\mathrm{TD}_{50}}{\mathrm{EUD}}\right] 4 \gamma_{50}}
$$

where $\mathrm{TD}_{50}$ is the dose at which probability of complication becomes $50 \%$ in 5 years, $\gamma_{50}$ is the slope of sigmoidal dose response curve of normal tissue at $50 \%$ complication probability. These tissue-specific parameters are based on Niemierko model [16].

NTCP of brainstem, spinal cord and both parotids was calculated for plan evaluation. V26 and V32 of each parotid were also calculated. Tolerance criteria of 70\% and 50\% were set for V26 and V32, respectively [13].

A unique quality index was generated by combing all above mentioned index numbers, taking a relative weight of each index numbers.

$\mathrm{CPQI}=\sum_{i}^{n} \sum_{j}^{m}\left(W_{i} X_{i}-W_{j} X_{j}\right)$

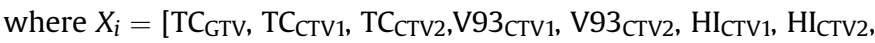
$\mathrm{TCP}_{\mathrm{GTV}}$ ], is the plan quality index (PQI) of targets (GTV and CTVs), and $X_{j}=\left[\mathrm{NTCP}_{\text {brainstem, }}, \mathrm{NTCP}_{\text {cord }}, \mathrm{NTCP}_{\text {ipsilateralparotid, }} \mathrm{NTCP}_{\text {con- }}\right.$ tralateralparotid, V26, V32], is the plan quality index of OARs. $W$ is the relative weight of each index. The relative weight was defined as the maximum value of the index divided by the sum of all the maximum values of the indices. For V26 and V32 of parotids, their maximum values were defined as the reciprocal of their tolerance criteria.

The philosophy of radiotherapy is to deliver as high as possible a dose to target while sparing the surrounding normal tissue as much as you can. For target quality index, the higher the value the better the plan quality, while for OAR quality index, the lower the value the better the plan quality. So for CPQI, the plan quality is better for a higher value.

\section{Validation}

Three methods were applied to assess the accuracy of this new plan quality index. Firstly, detailed statistical analysis on various plan quality indices, both the target indices and OAR indices, was conducted. Secondly, all the plans were scored and compared according to radiation oncology quality system (ROQS ${ }^{\mathrm{TM}}$ ) using a plan quality matrix (PQM) for benchmarking treatment planning and contouring [20]. In the original ROQS, there are 21 sub-matrices for head neck patients with a maximum score of 150 . Due to different prescription dose levels for target and different number of OARs involved in the inverse optimization, we picked 9 of the sub-matrices with a maximum score of 65 according to our prescription and the number of OARs involved. Thirdly, two senior radiation oncologists were asked to rank the plans with different OAR priority to further verify the accuracy and reliability of this new CPQI. Physician's evaluation was based on their clinical experience by visual inspecting the DVHs and isodose distribution on CT data slice by slice. Parameters of V93, V110, Dmax, Dmean from DVHs, as well as the coverage of $95 \%$ isodose line on each CT slices were employed by the physicians.

\section{Statistical methods}

Comparison of quality indices among plans with different OAR priority was analyzed with One way ANOVA method. All statistical analysis was conducted with SPSS 17.0 software. Difference was considered statistically significant if $P<0.05$ and the linear trend $F>1$.
Table 1

Index matrix calculated from the extracted DVH.

\begin{tabular}{lllllll}
\hline GTV & CTV1 & CTV2 & Brainstem & Cord & $\begin{array}{l}\text { Contra para } \\
\text { parotid }\end{array}$ & $\begin{array}{l}\text { Ipsilateral } \\
\text { parotid }\end{array}$ \\
\hline Dmax & Dmax & Dmax & Dmax & Dmax & Dmax & Dmax \\
Dmean & Dmean & Dmean & Dmean & Dmean & Dmean & Dmean \\
Dmin & Dmin & Dmin & EUD & EUD & EUD & EUD \\
EUD & EUD & EUD & NTCP & NTCP & NTCP & NTCP \\
TCP & TCP & TCP & V65 & V54 & V26 & V26 \\
V93 & V93 & V93 & & & V32 & V32 \\
V95 & V95 & V95 & & & & \\
V110 & V110 & V110 & & & & \\
V115 & V115 & V115 & & & & \\
\hline
\end{tabular}

Note: Dmax is the maximum dose, Dmean is the mean dose, Dmin is the minimum dose, V93 is the volume receiving 93\% of the prescribed dose, similar to V95, V110, V115; V65 is the volume that received 65 Gy dose, similar to V54, V26, and V32.

\section{Results}

The volume of GTV and CTVs varies according to different stages of the patients. The mean GTV volume of all these patients was about $23.32 \pm 17.42 \mathrm{cc}(4.03-55.87 \mathrm{cc})$; the mean volume of CTV1 and CTV2 was about $269.14 \pm 43.57 \mathrm{cc}(185.15-332.54 \mathrm{cc})$, and $138.07 \pm 18.23 \mathrm{cc}(104.45-167.30 \mathrm{cc})$, respectively. For each patient, four multi-isocenter IMRT plans were generated with different OAR priority, that was OAR low, OAR normal, OAR high, and PTV only, respectively. There were total of 40 plans for 10 patients.

Both cumulative DVH and differential DVH were extracted from TPS and reproduced by a custom software, as shown in Fig. 2. The extraction process copied the DVHs from TPS into text file then imported into the custom software, so the reproduced DVHs were identical to original ones. Cumulative DVH was used to read some of the plan quality indices directly. Differential DVH was used to calculate the dose-volume based items, such as Dmean, and EUD.

With extracted DVH data, Table 1 shows some of the matrix indices that had been calculated for plan filtering process and for plan quality evaluation with the custom software. A user defined filtering matrix can be easily setup by randomly selecting items from Table 1. For the criteria applied in this study, six plans failed to satisfy the PTV V93 < 1\%, two plans failed to satisfy V32 of ipsilateral parotid $<50 \%$. However, for the sake of data homogeneity, filtering criteria were adjusted to PTV V $90<1 \%$, parotid V $32<60 \%$.

Plan quality index values for targets, OARs and the composite plan quality index were summarized in Table 2. One-way ANOVA analysis with Turkey Post Hoc test indicated that there was no significant difference among groups of PTV PQI $(F=3.79$, $P=0.059)$. While there were significant differences among groups for OAR PQI $(F=49.0, p<0.01)$ and CPQI $(F=55.17, p<0.01)$. Table 2 demonstrated that of NPC SIB-IMRT, plans with high OAR priority may achieve less target coverage, while it can achieve a much better OAR sparing. For plans passed the filtering process, OAR high priority plan would be an optimal plan for clinical treatment.

To verify the results indicated by this new composite plan quality index, detailed statistical analysis on plan quality indices was conducted, as shown in Tables 3 and 4. As we can see, plans

Table 2

Plan quality index values for plans with different OAR constraints.

\begin{tabular}{lllr}
\hline \multirow{2}{*}{ Treatment plans } & & \multicolumn{2}{l}{ Plan quality index } \\
\cline { 3 - 4 } Constraint & Target PQI & OAR PQI & Composite PQI \\
\hline OAR low & $0.93 \pm 0.017$ & $0.71 \pm 0.081$ & $0.22 \pm 0.08$ \\
OAR normal & $0.92 \pm 0.016$ & $0.43 \pm 0.087$ & $0.49 \pm 0.077$ \\
OAR high & $0.90 \pm 0.019$ & $0.20 \pm 0.067$ & $0.71 \pm 0.062$ \\
PTV only & $0.92 \pm 0.022$ & $1.14 \pm 0.16$ & $(-) 0.21 \pm 0.16$ \\
\hline
\end{tabular}


Table 3

ANOVA analysis results for target and OAR quality indices for plans with different OAR constraints.

\begin{tabular}{|c|c|c|c|c|c|c|}
\hline & OAR low & OAR normal & OAR high & TPV only & $F$ & $\operatorname{sig}(p)$ \\
\hline \multicolumn{7}{|l|}{ GTV } \\
\hline Dmin & $59.53 \pm 2.01$ & $59.19 \pm 1.71$ & $58.13 \pm 2.30$ & $60.31 \pm 2.05$ & 0.20 & 0.66 \\
\hline Dmax & $74.20 \pm 0.85$ & $74.03 \pm 0.94$ & $74.54 \pm 1.72$ & $73.86 \pm 1.25$ & 0.08 & 0.78 \\
\hline Dmean & $68.41 \pm 0.69$ & $68.26 \pm 0.40$ & $68.18 \pm 0.55$ & $68.06 \pm 0.58$ & 2.00 & 0.17 \\
\hline TCP & $0.94 \pm 0.0086$ & $0.94 \pm 0.009$ & $0.94 \pm 0.012$ & $0.93 \pm 0.012$ & 2.68 & 0.11 \\
\hline \multicolumn{7}{|l|}{ CTV1 } \\
\hline Dmin & $47.66 \pm 2.14$ & $44.91 \pm 0.91$ & $40.21 \pm 3.64$ & $48.38 \pm 1.40$ & 0.45 & 0.51 \\
\hline Dmax & $70.67 \pm 0.98$ & $70.61 \pm 1.41$ & $70.90 \pm 1.24$ & $71.12 \pm 0.70$ & 1.06 & 0.31 \\
\hline Dmean & $55.71 \pm 0.28$ & $55.61 \pm 0.28$ & $55.44 \pm 0.31$ & $55.44 \pm 0.29$ & 6.03 & 0.019 \\
\hline $\mathrm{HI}$ & $0.942 \pm 0.028$ & $0.943 \pm 0.027$ & $0.945 \pm 0.025$ & $0.944 \pm 0.028$ & 0.04 & 0.84 \\
\hline \multicolumn{7}{|l|}{ CTV2 } \\
\hline Dmin & $49.78 \pm 2.00$ & $48.27 \pm 2.17$ & $44.30 \pm 3.40$ & $49.62 \pm 1.65$ & 1.75 & 0.20 \\
\hline Dmax & $62.44 \pm 4.51$ & $62.55 \pm 4.29$ & $62.83 \pm 4.31$ & $62.44 \pm 4.80$ & 0.002 & 0.96 \\
\hline Dmean & $55.31 \pm 0.54$ & $55.01 \pm 0.25$ & $54.87 \pm 0.20$ & $54.89 \pm 0.33$ & 7.71 & 0.009 \\
\hline $\mathrm{HI}$ & $0.995 \pm 0.0069$ & $0.997 \pm 0.0042$ & $0.997 \pm 0.0055$ & $0.996 \pm 0.0065$ & 0.009 & 0.92 \\
\hline
\end{tabular}

with high OAR priority showed less GTV and CTVs coverage. However, this inferior coverage was not statistically significant, except for the mean dose (Dmean) of CTV1 $(p=0.02)$ and CTV2 $(p=0.01)$. On the contrary, the OAR sparing achievement for plans with high OAR priority was of significant difference except for the volume of ipsilateral parotid receiving $26 \mathrm{~Gy}$ (V26). This result was consistent with our CPQI indication.

The plan quality matrix scoring results were shown in Table 5. The maximum score was achieved for OAR high plans with a mean around 40 out of 65 . The total score increases with the priority of OAR. This was comparable to our CPQI results. The independent target and OAR scores were also consistent with the CPQI results.

Physician ranking were conducted by two senior radiation oncologists independently. The evaluation result of these two physicians was quite similar. No plans from PTV only and OAR low were accepted due to inferior OAR sparing and target coverage. OAR high plans were selected in 9 patients. Only in one patient that OAR normal plan was selected. The choice ratio for OAR high plans for all these 10 patients was about $90 \%$. This was also consistent with CPQI results.

\section{Discussion}

Radiotherapy treatment plan evaluation is commonly based on 3D dose distribution and its 1D representation, DVH.
Radiobiological ranking by calculating EUD, TCP and NTCP is also widely applied $[5,21]$. However, radiobiological indices are usually used as complement to dosimetric evaluation, calculated separately as an additional tool for plan evaluation. A composite plan quality index was suggested in this study to integrate both dosimetric and radiobiological quality indices.

Target quality indices indicated there was no significant difference on target coverage for SIB-IMRT plans with different OAR priority. There was a slight trend that Dmin, Dmax, Dmean and HI of targets increased when the OAR priority decreased from high to low, but with no statistical significance. This result was different from IMRT plans with different OAR priority. Generally, a decrease in target coverage and an increase in HI with the increase importance of OAR weighting were expected, as had been reported for IMRT plans [22]. This indicated for SIB IMRT plans, with the introduction of multiple level dose prescriptions, the planning and optimization have become more complex. One other possible source of this complexity could be the multi-isocenter planning strategy employed with a small MLC. The OAR quality indices were consistent with expectation. With the increase of OAR weight, better OAR sparing was achieved.

Some of the items in the filtering matrix is more general for filtering purpose. It's hard to normalize them, such as Dmax, Dmean. So the composite plan quality index did not combine all the target and OAR indices, but rather pick some of them that are easy

Table 4

ANOVA analysis results for OAR quality indices for plans with different OAR priority.

\begin{tabular}{|c|c|c|c|c|c|c|}
\hline OAR & OAR low & OAR normal & OAR high & TPV only & $F$ & $\operatorname{sig}(p)$ \\
\hline \multicolumn{7}{|l|}{ Brainstem } \\
\hline $\operatorname{Dmax}(\mathrm{Gy})$ & $52.19 \pm 2.89$ & $47.38 \pm 2.39$ & $44.13 \pm 1.51$ & $58.51 \pm 2.92$ & 18.62 & $<0.01$ \\
\hline Dmean (Gy) & $31.91 \pm 2.23$ & $28.52 \pm 1.72$ & $24.97 \pm 1.25$ & $36.98 \pm 4.14$ & 10.22 & $<0.01$ \\
\hline $\operatorname{NTCP}\left(\times 10^{-4}\right)$ & $28.41 \pm 15.41$ & $7.58 \pm 3.72$ & $3.17 \pm 1.25$ & $42.40 \pm 6.02$ & 38.12 & $<0.01$ \\
\hline \multicolumn{7}{|l|}{ Spinal cord } \\
\hline Dmax & $47.38 \pm 2.38$ & $40.82 \pm 1.31$ & $38.14 \pm 0.77$ & $59.36 \pm 2.81$ & 38.12 & $<0.01$ \\
\hline Dmean & $35.51 \pm 1.51$ & $31.02 \pm 0.69$ & $29.04 \pm 0.76$ & $44.31 \pm 2.33$ & 139.83 & $<0.01$ \\
\hline $\operatorname{NTCP}\left(\times 10^{-4}\right)$ & $5.37 \pm 7.26$ & $0.40 \pm 0.20$ & $0.13 \pm 0.05$ & $198.44 \pm 110.16$ & 55.00 & $<0.01$ \\
\hline \multicolumn{7}{|l|}{ Ipsilateral parotid } \\
\hline Dmax & $52.86 \pm 1.65$ & $46.98 \pm 3.89$ & $40.82 \pm 4.70$ & $59.08 \pm 2.54$ & 7.33 & 0.01 \\
\hline Dmean & $33.85 \pm 1.59$ & $27.62 \pm 1.37$ & $22.95 \pm 1.76$ & $40.87 \pm 2.33$ & 41.58 & $<0.01$ \\
\hline V26 & $81.10 \pm 5.21$ & $66.10 \pm 5.66$ & $41.20 \pm 12.36$ & $92.69 \pm 7.18$ & 0.74 & 0.4 \\
\hline V32 & $68.70 \pm 6.19$ & $41.70 \pm 7.65$ & $17.77 \pm 8.02$ & $85.31 \pm 7.29$ & 6.27 & 0.02 \\
\hline $\operatorname{NTCP}\left(\times 10^{-4}\right)$ & $76.97 \pm 59.89$ & $3.33 \pm 2.69$ & $0.18 \pm 0.18$ & $1130.19 \pm 729.97$ & 37.15 & $<0.01$ \\
\hline \multicolumn{7}{|c|}{ Contralateral parotid } \\
\hline Dmax & $45.30 \pm 3.26$ & $38.30 \pm 3.66$ & $32.59 \pm 4.20$ & $53.93 \pm 2.87$ & 16.21 & $<0.01$ \\
\hline Dmean & $27.05 \pm 1.87$ & $21.43 \pm 1.84$ & $17.15 \pm 1.52$ & $34.46 \pm 2.64$ & 39.86 & $<0.01$ \\
\hline V26 & $59.67 \pm 9.30$ & $33.69 \pm 12.94$ & $11.57 \pm 5.80$ & $85.05 \pm 12.23$ & 13.36 & $<0.01$ \\
\hline V32 & $36.71 \pm 10.10$ & $12.57 \pm 8.43$ & $1.86 \pm 1.98$ & $69.50 \pm 13.88$ & 41.58 & $<0.01$ \\
\hline $\operatorname{NTCP}\left(\times 10^{-4}\right)$ & $4.44 \pm 3.46$ & $0.11 \pm 0.09$ & $0.006 \pm 0.0049$ & $303.08 \pm 307.55$ & 16.97 & $<0.01$ \\
\hline
\end{tabular}


Table 5

Plan quality matrix score analysis results.

\begin{tabular}{lrllrrc}
\hline & \multicolumn{1}{c}{ OAR low } & OAR normal & OAR high & \multicolumn{1}{c}{ PTV only } & \multicolumn{1}{c}{$F$} & sig $(p)$ \\
\hline PTV score & $25.7 \pm 2.5$ & $26.2 \pm 0.6$ & $25.5 \pm 2.4$ & $26.6 \pm 0.9$ & 0.61 & 0.44 \\
OAR score & $7.5 \pm 4.1$ & $13.4 \pm 0.9$ & $13.6 \pm 0.8$ & $0.5 \pm 1.6$ & 41.47 & $<0.001$ \\
Total score & $33.2 \pm 5.7$ & $39.8 \pm 0.6$ & $40.0 \pm 0.9$ & $27.1 \pm 1.7$ & 17.97 & $<0.001$ \\
\hline
\end{tabular}

to be normalized and weighted. As a linear combination of related plan quality indices, the composite plan quality index is simple and straightforward. And it can be easily adapted for different tumor sites and protocols. One potential problem of this linear combination would be that one composite value could be as a result of different combinations. One solution to eliminate the effect of this potential problem is to introduce a filtering process. As qualified plans passed the filtering process, the potential possibility of the composite index value resulted from different combinations has little effect on the plan quality.

The result of CPQI was consistent with detailed statistical analysis of plan quality indices and plan quality matrix scoring results of ROQS. With the increased weight of OAR priority, there were much better OAR sparing effects, resulting in the highest CPQI score for plans with high OAR priority. This result was also consistent with physician's ranking result, in which about $90 \%$ of the choice are plans with OAR high priority. There was one patient with normal OAR priority was preferred by both physicians instead of OAR high plan. The reason was that there was a cold spot near GTV region for OAR high plan. This result demonstrated one week side of this new plan quality index. It lacks spatial information of the dose distribution. This is also an intrinsic limitation of DVH based plan quality indices. To compensate for the lack of spatial information within the $\mathrm{DVH}$, various supplemental methods had been investigated [23] However, none of these supplemental presentations had been accepted widely for clinical use due to the complexity, less intuitive than the simple DVH concept, or its limited spatial information available [6]. Therefore, to integrate some spatial information into our new plan quality index still needs further efforts. The application of this new plan quality index in other treatment modality and other cancer patients also needs further study.

\section{Conclusion}

A new composite plan quality index integrating both dosimetric and radiobiological indices was proposed in this study to facilitate the evaluation and comparison of SIB-IMRT plans for nasopharyngeal cancer patients. The introduction of a filtering process can filter some unqualified plans and save evaluation time. The accuracy of this CPQI was verified by detailed statistical analysis, ROQS scoring results and physician's ranking results. This is a promising evaluation tool for optimal plan selection for NPC SIB IMRT.

\section{Conflict of interest statement}

There are no conflict of interests for this manuscript.

\section{Acknowledgments}

The paper was supported by Wenzhou Science and Technology Bureau Funding (Y20120137) and the Scientific Research Foundation for the Returned Overseas Chinese Scholars (604090656/ 037).

\section{References}

[1] Lee N, Xia P, Quivery JM, Sultanem K, Poon I, Akazawa C, et al. Intensitymodulated radiotherapy in the treatment of nasopharyngeal carcinoma: an update of the UCSF experience. Int J Radiat Oncol Biol Phys 2002;53:12-22.

2] Kam MK, Teo PM, Chau RM, Cheung KY, Choi PH, Kwan WH, et al. Treatment of nasopharyngeal carcinoma with intensity-modulated radiotherapy: the Hong Kong experience. Int J Radiat Oncol Biol Phys 2004;60:1440-50.

[3] Mohan R, Wu QW, Manning M, Schmidt-Ullrich R. Radiobiological considerations in the design of fractionation strategies for intensity-modulated radiation therapy of head and neck cancers. Int J Radiat Oncol Biol Phys 2000;46: 619-30.

[4] Feuvret L, Noel G, Mazeron JJ, Bey P. Conformity index: a review. Int J Radiat Oncol Biol Phys 2006;64:333-42.

[5] Leung TLH, Kan MWK, Cheng ACK, Wong WKH, Yau CC. A new dose-volumebased plan quality index for IMRT plan comparison. Radiother Oncol 2007;85: 407-17.

[6] Zhao B, Joiner MC, Orton CG, Burmeister J. "SABER": a new software tool for radiotherapy treatment plan evaluation. Med Phys 2010;37:5586-92.

[7] Wu S, Xie C, Jin X, Zhang P. Simultaneous modulated accelerated radiation therapy in the treatment of nasopharyngeal cancer: a local center's experience. Int J Radiat Oncol Biol Phys 2006;66:S40-6.

[8] Jin X, Wu S, Yu J, Yi J. Technique and dosimetric consideration in multiisocenter Intensity modulated radiotherapy for nasopharyngeal carcinoma with small multileaf collimator. Med Dosim 2009;34:9-15.

[9] Llacer J, Solberg TD, Promberg C. Comparative behavior of the dynamically penalized likelihood algorithm in inverse radiation therapy planning. Phys Med Biol 2001;46:2637-63.

[10] Llacer J. Inverse radiation treatment planning using the dynamically Penalized likelihood method. Med Phys 1997;24:1751-64.

[11] Drzymal RE, Harms WB, Purdy JA. Dose-volume histograms for 3D radiation treatment plans. Med Phys 1987;14:460.

[12] Austin-Seymour MM, Chen GTY, Castro JR, Saunders WH, Pitluck S, Woodruff $\mathrm{KH}$, et al. Dose-volume histogram analysis of liver radiation tolerance. Int J Radiat Oncol Biol Phys 1986;12:31-5.

[13] Emami B, Lyman J, Brown A, Coia L, Goitein M, Munzenrider JE, et al. Tolerance of normal tissue to therapeutic irradiation. Int J Radiat Oncol Biol Phys 1991;21(1):109-22.

[14] Lee N, Garden A, Kramer A, Xia P. A phase II study of intensity modulated radiation therapy (IMRT) $+/-$ chemotherapy for nasopharyngeal cancer. RTOG Protoc 2005;0225.

[15] Wambersie A, Landber T. ICRU report 62: prescribing, recording, and reporting photon beam therapy [Supplement to ICRU Report 50]. Bethesda: ICRU; 1999.

[16] Niemierko A, Goiten M. Modeling of normal tissue response to radiation critical volume model. Int J Radiat Oncol Biol Phys 1993;25:135-45.

[17] Burman C, Kutcher GJ, Emami C, Goiten M. Fitting of normal tissue tolerance data to an analytic-function. Int J Radiat Oncol Biol Phys 1991;21:123-35.

[18] Niemierko A, Goiten M. Implementation of a model for estimating TCP for an inhomogeneously irradiated tumor. Radiother Oncol 1993;29:140-7.

[19] Okunieff P, Morgan D, Niemierko A, Suit HD. Radiation dose-response of human tumors. Int J Radiat Oncol Biol Phys 1995;32:1227-37.

[20] Nelms BE, Robinson G, Markham J, Velasco K, Boyd S, Narayan S, et al. Variation in external beam treatment plan quality: an inter-institutional study of planners and planning systems. Pract Radiat Oncol 2012:2:296-305.

[21] Pyakuryal A, Myint WK, Gopalakrishnan M, Jang S, Loqemann JA, Mittal BB. A computational tool for the efficient analysis of dose-volume histograms for radiation therapy treatment plans. J Appl Clin Med Phys 2010;11:137-58.

[22] Grzadziel A Grosu AL, Kneschaurek P. Three-dimensional conformal versus intensity-modulated radiotherapy dose planning in stereotactic radiotherapy: application of standard quality parameters for plan evaluation. Int J Radiat Oncol Biol Phys 2006;66:S87-94.

[23] Cheng CW, Das IJ. Treatment plan evaluation using dose-volume histogram (DVH) and spatial dose-volume histogram (zDVH). Int J Radiat Oncol Biol Phys 1999;43:1143-50. 